\title{
PREVENCIÓN DEL DELITO Y CONSTRUCCIÓN DE FEMINIDADES JUVENILES
}

\section{CRIME PREVENTION AND YOUTHFUL FEMININITY CONSTRUCTION}

\author{
Marina Medan*
}

RESUMEN

Este trabajo articula las dimensiones de edad y género en la construcción de beneficiarias en un programa de prevención del delito juvenil implementado en el Gran Buenos Aires, Argentina. Se identifica el tratamiento que este dispositivo estatal destinado a jóvenes $-y$ pensado para varones - realiza sobre las chicas. La legitimación institucional de necesidades de las beneficiarias condiciona la configuración de feminidades y restringe aspectos de la experiencia juvenil y femenina.

PALABRAS CLAVE: ARGENTINA * PROGRAMAS DE AYUDA * PREVENCIÓN DEL DELITO * JUVENTUD * GÉNERO $*$ EDAD

\section{ABSTRACT}

This article seeks to articulate age and gender dimensions within the female client construction in a crime prevention program, implemented in Gran Buenos Aires, Argentine. It identifies how girls are managed in a state intervention, targeted to youth people but specially on boys. The institutional legitimacy of female needs has an influence on the way girls configure their gender identity and on their own young and female experience.

KEYWORDS: ARGENTINE * AID PROGRAMMES * CRIME PREVENTION * YOUTH * GENDER * AGE

1 Este trabajo revisa y retoma algunos argumentos expuestos en una presentación realizada en el XXVIII Congreso Internacional ALAS, en septiembre de 2011.

* $\quad$ Facultad de Ciencias Sociales de la Universidad de Buenos Aires (UBA), Argentina. marinamedan@gmail.com 


\section{INTRODUCCIÓN}

Este trabajo articula las dimensiones de edad y género en la construcción de beneficiarias en un programa de prevención del delito juvenil implementado en el Gran Buenos Aires, Argentina.

Este artículo se ubica en las discusiones sobre la construcción social del par inclusión/ exclusión, y sobre los modos de gobernar la juventud en riesgo desde una perspectiva constructivista ${ }^{2}$ para analizar los programas sociales $y$ el rol del Estado.

Los programas sociales dirigidos a la infancia, adolescencia y juventud suelen definir su población destinataria otorgando centralidad a la dimensión etaria, a la que agregan condicionalidades de clase, étnia o de género ${ }^{3}$. Asimismo, muchas de las intervenciones dirigidas a esta población, tienen entre sus principales objetivos que los y las jóvenes logren construir un "proyecto de vida". Este objetivo sugiere que estas poblaciones no pueden hacerlo por sí solas y que necesitan asistencia para concretarlo o que si acaso pueden hacerlo, la ayuda institucional redundará en que el proyecto de vida que gesten, sea el "deseable".

Si bien, la gestación de un "proyecto de vida" aparece vinculada con trayectorias educativas sostenidas, inserciones laborales estables y la conformación de la familia propia, la investigación empírica de la que surge este

2 Este artículo entiende a los programas estatales como dispositivos que representan y exceden al Estado. Esto requiere dos aclaraciones. Por un lado, el Estado al que representan es entendido aquí como una entidad multifacética y conformada en capas, compuesta por diferentes aparatos que pueden operar de forma inconsistente (Haney, 1996). Los programas, a su vez, lo exceden ya que logran su forma final en sus implementaciones localizadas en espacios y tiempos específicos, en las que sus diseños programáticos entran en interacción cotidiana, mediados por agentes estatales, con sus beneficiarios(as).

Con respecto a esta última dimensión, el género, las definiciones de destinatarios suelen no pronunciarse; esto supone que - en principio- admiten chicas y varones; en algunos pocos casos hay programas destinados a mujeres jóvenes -en general vinculados con temas de salud sexual y reproductiva- y no tenemos constancia de que existan programas destinados solo a varones. trabajo evidencia que en el marco de programas sociales, este no tiene las mismas características según se trate de chicas o varones. Esto debido a que los programas, no solo colaboran con la construcción social y el procesamiento de las edades de las personas, sino porque portan ideas sobre el género y sobre qué aspectos deberían caracterizar unos proyectos de vida deseables para chicas y varones.

Cuando chicas $y$ varones se encuentran con los programas sociales, se ponen en diálogo las expectativas $y$ activos de ambas partes. Desde unas prácticas que suponen un ejercicio diferencial de poder — en tanto los(as) agentes que implementan los programas deciden si una persona es admitida $y$ mantenida dentro del dispositivo- los programas evalúan el modo en que las chicas y los varones se acercan a los ideales previstos; asimismo, discuten o legitiman dichas acciones, construyendo modos legítimos de feminidades y masculinidades jóvenes ${ }^{4}$.

\section{LAS LUPAS TEÓRICAS}

Existe consenso en que las intervenciones institucionales (ya sean estatales, religiosas, privadas, etc.) contribuyen con sus definiciones de actores $y$ objetivos, a la organización y caracterización de distintos grupos de personas, con fines de regulación social. Valeria Llobet (2009) sostiene que las instituciones cumplen un papel legislador, al señalar las cláusulas bajo las que, por ejemplo, la inclusión social y cultural podrá darse, incluyendo el proceso de adquisición y reconstrucción de características típicas, el manejo de las relaciones sociales y su transformación en posiciones subjetivas singulares. En este sentido, los programas, desde sus diseños hasta sus implementaciones, interpretan necesidades de sus destinatarios, definen satisfactores para estas $y$ legitiman demandas que coinciden con esas

$4 \quad$ La focalización en la generación y el género no supone invisibilizar los condicionantes de clase o étnicos. La condición socioeconómica desaventajada a la que pertenecen los(as) jóvenes que protagonizan el análisis, atraviesa el mismo. Sobre este tema pueden consultarse Medan (2012, en prensa). La condición étnica en este estudio, no se presentó como una variable significativa. 
interpretaciones; aún concibiendo dentro de ellos procesos heterogéneos y no siempre coherentes, se establecen como la voz autorizada para definir las reglas del juego, los posibles $y$ los impensables (Fraser, 1991).

Cuando las poblaciones a regular son las nuevas generaciones, la tarea legislativa es aún más sensible. De lo que se trata, no es solamente de garantizar el orden sino la reproducción social (Llobet, 2009). En ese punto, la condición de la transición entre la juventud y la adultez cobra un espacio clave. Así puede entenderse la preocupación de los programas sociales por encaminar transiciones "deseables" hacia la adultez, a través de la gestación del "proyecto de vida”. Esta expresión requiere ser recontextualizada en cada caso para moderar sus polémicos matices (Llobet, 2009).

Asimismo, al prescribir la necesidad de un "proyecto de vida", los programas caracterizan a los(as) jóvenes como sujetos carentes (Chaves, 2005) y ubicados(as) en una posición de menor jerarquía y evolución respecto de ciertos parámetros adultocráticos, en torno a la conformación plena de una persona. La juventud se dibuja como una etapa incompleta $y$ de transición a un estado que sí goza de plenitud como sería la adultez.

En esa preocupación transicional, la edad parece ser la dimensión a la que apelan los programas sociales para comenzar a definir sus destinatarios(as). Sin embargo, tal como puntualizó Bourdieu (1990), es sabido que las edades son arbitrarias $y$ que se refieren a una de las formas en que se representa la lucha por el poder, en este caso, entre unas personas más jóvenes y otras más viejas. A partir de esas luchas resultarán las fronteras provisorias, que intentarán establecer cierto orden, por ejemplo, entre quiénes, jóvenes o adultos, acceden a tales bienes, tales usos de la palabra autorizada, tales espacios. Asimismo, en la misma operación quedarán denegados otros. La edad es, por tanto, una categoría relacional, tanto en su dimensión generacional como respecto de otras. Cuando las edades biológicas se cruzan con condiciones de clase, con accesos desiguales a bienes materiales $y$ simbólicos, $y$ con pertenencias étnicas y de género, las historias que se configuran pueden resultar verdaderamente disimiles. Así, resulta más útil pensar que las etapas vitales de las personas no se componen por un conjunto fijo de características homogéneas que se rigen por compartir el año de nacimiento, sino que son más bien flexibles y que se constituyen $y$ moldean en cada persona a partir de la interrelación de múltiples dimensiones y factores, entre las cuales la clase social en cruce con el género cobra una importancia considerable.

Ahora bien, así como, la definición y el procesamiento de las edades son relacionales, la dimensión del género - es decir, la construcción social de la diferencia sexual- también lo es. Eleonor Faur (2004: 47-53) sugiere que la tensión entre la elección individual de los sujetos y la coerción social del entorno es una de las que permite pensar la construcción social del género, por ejemplo, dentro de programas sociales. Estos son parte del entorno disponible y a su vez, encaran acciones con ideas y representaciones sobre el género (Guzmán, 2002), aunque parezcan presentarse como neutrales. Los programas actúan desde posiciones de género a partir de que sus trabajadores(as) son sujetos imbuidos en el género, tienen sus percepciones sobre modelos deseables de ser varones o chicas jóvenes, por lo tanto, bajo esas ideas implementan los planteamientos institucionales. En síntesis, las instituciones encarnan algunas y no otras, de las representaciones circulantes sobre lo masculino y lo femenino como regulaciones normativas (Scott, 2000) y a su vez, las identidades de género de las personas "no responden meramente a elecciones personales ni exclusivamente a formatos construidos en el orden social" (Faur, 2004: 52).

El marco teórico expuesto sugiere que los programas inciden con sus nominaciones $y$ propuestas en las relaciones entre jóvenes y adultos, $y$ entre hombres y mujeres, en síntesis: modelando relaciones deseables de género y edad. Ahora bien, cuando las personas quieren recibir la asistencia de los programas, ponen en discusión sus modos de vida cotidiana ${ }^{5}$ con

5 Como se señaló, esos modos de vida no fueron configurados de forma autónoma, sino de forma relacional. De hecho, las personas nacieron, se criaron 
las expectativas institucionales sobre ellos $y$ ellas en función de su pertenencia a una clase social, a una posición de género o en la etapa del ciclo vital. Si bien, la discusión que mantienen ambas partes no supone prácticas equipotenciales, existe un espacio de contienda (Fraser, 1991) en el que grupos dominantes y subordinados negocian las condiciones de la asistencia.

En este artículo, interesa recuperar ese proceso de construcción negociado de feminidad al interior del programa $y$ ahondar en las consecuencias que conlleva.

\section{REFERENTE EMPÍRICO Y METODOLOGÍA}

El referente empírico de este trabajo es un estudio de caso sobre una implementación de un programa estatal de prevención del delito juvenil en un barrio del Gran Buenos Aires, Argentina, durante 2008-2009. La dinámica de implementación del programa consta de reuniones semanales de dos horas entre el equipo técnico y los(as) jóvenes en distintos espacios del barrio. Las reuniones incluyen talleres sobre el eje "vincular" (relacionado con el proyecto de vida), el "mundo del trabajo" (reinserción escolar y laboral), "jurídico" (fortalecimiento de derechos y resolución de causas penales) y "sociocomunitario". Este programa incluye una transferencia condicionada de ingresos a los(as) beneficiarios(as) de $\$ 150$ mensuales, equivalente a us $\$ 36$.

Durante el período en que se obtuvieron los datos, el grupo de beneficiarios(as) se compuso de 31 varones y 15 mujeres, todos(as) argentinos(as), con un promedio de edad grupal de 22 años. Todas las chicas eran madres o estaban embarazadas y solo un tercio de los varones tenían hijos(as) (aunque no todos los tenían a cargo). Algunos de los beneficiarios(as) habían cometido delitos, otros(as) tenían prácticas delictivas o estaban, según criterios del programa, en riesgo de hacerlo. El equipo técnico estaba compuesto por una agente estatal trabajadora social (32 años) y un estudiante de

y crecieron en el seno de instituciones (la familia, el mercado, la escuela, los medios de comunicación, etc.) cuyas ideas sobre la edad y el género han permeado sus vidas. comunicación (31 años) que no vivían dentro del barrio, además de una operadora comunitaria (34 años), habitante del barrio. Todos los sueldos estaban a cargo del Municipio.

Para dar cuenta del modo en que se construyen las feminidades juveniles en el seno de este programa se analizaron: los materiales obtenidos en la observación participante de las interacciones entre agentes $y$ beneficiarias(os) en 76 encuentros, $y$ de entrevistas en profundidad a ocho jóvenes $y$ tres agentes estatales, así como, documentos institucionales ${ }^{6}$. En los materiales se rastrearon: condiciones de acceso, permanencia y egreso del programa, expectativas sobre los impactos del programa en la vida de las y los jóvenes, logros y dificultades en la obtención de los resultados previstos, instancias de negociación entre oferta y demanda, así como, consecuencias de las negociaciones entre partes. Para este trabajo se puso especial atención en la mirada hacia las relaciones generacionales, de género y clase social observadas en las interacciones entre las(os) agentes del programa y las chicas.

\section{DESARROLLO}

\section{LOS BENEFICIARIOS Y LAS BENEFICIARIAS SEGÚN LAS DEFINICIONES INSTITUCIONALES}

Según los documentos institucionales, este programa de prevención del delito está destinado a adolescentes y jóvenes de entre 13 y 25 años que viven en situación de vulnerabilidad social $^{7}$ :

6 Se han cambiado los nombres de los informantes para proteger su identidad.

$7 \quad$ Según los documentos del programa, las variables que caracterizan la situación de vulnerabilidad son, entre otras: contacto cotidiano con violencia en sus múltiples formas, débiles o nulas inserciones educativas y laborales, incitación a consumo de sustancias psicoactivas ilegales, acceso a armas a bajo costo, pasan muchas horas del día sin actividades organizadas ni cuentan con espacios de contención, aspiran a bienes de consumo caracterizados como propios de la etapa juvenil pero no tienen dinero para adquirirlos, son padres y madres tempranamente, sin recursos suficientes para formar un hogar propio, su vida cotidiana trascurre dentro de los límites del barrio, están vinculados a personas 
... en conflicto con la ley o en riesgo de estarlo (...) haber infringido una norma del código penal y no estar en situación de encierro; estar o haber estado involucrado en prácticas violentas próximas al delito; haber estado detenido en comisarías, institutos de menores $y / 0$ instituciones penitenciarias; haberse visto involucrado en detenciones preventivas en comisarías (Dirección Nacional de Protección Civil-DNPC, 2007).

La agente que lo implementa - haciéndose eco de lo arbitrario de las edades - señala que son considerados jóvenes quienes carecen de un "proyecto de vida", que aún no lo han podido "armar" y que por lo tanto, son inmaduros o están incompletos. Si acaso las incursiones delictivas se presentan como un proyecto de vida, el punto problemático es que no han podido diseñar uno deseable. De allí que el objetivo del programa sea "colaborar en que los jóvenes construyan un proyecto de vida alternativo al delito" como paso necesario de la transición a la adultez.

A su vez, lo que determina que ciertos(as) jóvenes puedan ser beneficiarios(as) es:

El grupo en riesgo, (para) trabajar la cuestión primaria de prevención del delito. Son pibes que capaz están cartoneando, juntándose con los de la esquina, la mitad de la familia delinquió, y viene la madre o un pariente o ellos mismos, por la motivación a salir a robar. 0 están en pequeñas acciones, $y$ capaz no tienen causas, no cometieron delitos grandes, pero están en pequeñas actividades, o que afanan guita en la casa, o que participa como cómplice de campana en alguna situación delictiva pero todavía no se metió y está ahí (Agente estatal, 2008).

Hasta este punto, la imagen sobre los(as) beneficiarios(as) se construye enfatizando en la dimensión de la edad y su procesamiento

que habitualmente cometen delitos, para ellos y ellas arriesgar la vida y la libertad forma parte de "las reglas del juego" (DNPC, 2007). social, así como, la clase y/o los condicionantes materiales. Sin embargo, en las observaciones de campo se verificó que el programa incluía chicas, aunque hubiera sido pensado para varones ${ }^{8}$. ¿El "proyecto de vida alternativo" o el vínculo con el riesgo, sería el mismo para chicas y chicos que compartían un mismo rango etario? En búsqueda de la respuesta, se indagó sobre las diferencias de género al momento de admitir beneficiarias.

Entrevistadora: —¿El Programa prevé admitir chicas que no han cometido delitos pero que al ser madres están en situación de vulnerabilidad?

Agente: - No, el perfil es el mismo, o que estén en riesgo, el marido, el cuñado, están todos presos ella está sola, y no tiene ingresos en la casa y está empezando a participar de alguna manera en la dinámica delictiva del barrio. Por eso se la incorpora, aunque no haya cometido delitos.

Las chicas serían más vulnerables a la comisión de delitos si no eran acompañadas por varones proveedores de ingresos; su riesgo era estar "solas" (de hombres); no aparecen otras figuras - madres, hermanas, amigas, o ellas mismas- que pudieran compensar esa carencia. El riesgo de los varones no suponía ausencia de chicas, sino necesidad de ingresos o influencia de pares. Esta posición "en riesgo" de las chicas, habilitaba, al menos enunciativamente, el delito femenino. Sería cuestión de indagar en el campo si efectivamente se producía por ausencia de varones proveedores.

$8 \quad$ La focalización se insinúa en los documentos institucionales (se señala a los varones jóvenes como los que encabezan las estadísticas delictivas, DNPC, 2007), en las caracterizaciones de los destinatarios ideales (ver Medan, 2010) y se practica en la implementación estudiada (se explicitará luego). Es más, el creador del programa a nivel nacional confirma que originalmente el programa se pensó para varones, más allá de lo finalmente haya sucedido (entrevista realizada para una instancia posterior de la investigación, al Director Nacional de Política Criminal, el 7/06/2011). 
Sin embargo, la observación de la dinámica en el trabajo de campo, sorprendió arrojando otros datos: por razones no estudiadas aquí y en coincidencia casual con el inicio del trabajo de campo, aumentó significativamente la cantidad de chicas admitidas que no tenían efectivas relaciones con el delito pero sí hijos ${ }^{9}$. Esta inesperada situación, requirió formular otras preguntas ¿qué relaciones entre el delito y las chicas jóvenes son posibles?, ¿cómo se delínea el proyecto de vida alternativo al delito para ellas?, ¿qué condiciones habilitan su transición a la adultez? y finalmente, el riesgo que aquejaba a las beneficiarias - y por lo cual había que intervenir institucionalmente- ¿era la comisión de delitos u otra cosa?

\section{LAS CHICAS, AVALADAS Y SUBORDINADAS}

De las 15 beneficiarias que tuvo el programa durante el trabajo de campo en el que se basa este artículo, solo 3 fueron admitidas por sus explícitas relaciones con el delito (una había estado en prisión y dos estaban imputadas, esperando el juicio oral). El resto se presentaron argumentando necesidades materiales $y$ dificultades de otras índoles, vinculadas al cuidado de sus hijos. ¿En qué lugar quedaba el delito femenino en este esquema? Cuando se la entrevistó, la agente explicó que dicha práctica estaba menos legitimada en el barrio que su versión masculina:

Hay una cuestión de aceptación, porque creo que hay muchas mujeres que delinquen en el barrio pero hay más aceptación de que lo haga el varón a que lo haga la mujer, entonces creo que también se juega la vergüenza, la representación barrial... (Operadora comunitaria, 2008).

En línea similar, las beneficiarias admitieron que el delito no era una actividad exclusivamente masculina, pero que a diferencia de

9 Según los testimonios y materiales institucionales, desde el inicio de la implementación en 2001 hasta 2008, el promedio de chicas era de 1 cada 7 varones. Desde 2008, la proporción aumentó y se registraron promedios de 1 chica cada 3 varones. los hombres, las mujeres eran "mal vistas" en el barrio por dedicarse a esas tareas.

Entrevistadora: - A las chicas que afanan ¿se las mira mal, peor que a los varones?

Beneficiaria: —Sí, sí ‘cómo una mujer?, ¿a vos te parece?, ¿no piensa en su mamá, en su papá?' a los varones no, y sí... pero a las mujeres se dice y ‘cómo si tiene hijos, hijas, cómo va a ser el día de mañana?' Y sí, la gente piensa así como te estoy diciendo... (Victoria, 31 años).

En síntesis, el delito femenino era parte del paisaje, pero de un paisaje en las sombras. Una sombra que llegaba hasta las puertas de acceso al programa. No se puede establecer si las chicas evitaban sus eventuales vínculos con el delito porque este estaba "mal visto" o porque otras argumentaciones les garantizaban la misma ayuda con menos descrédito. Pero lo cierto es que, independientemente de sus relaciones con el delito, elegían presentarse en función de su condición de madre. En los siguientes ejemplos, se observa cómo se presen$\tan$ las aspirantes a beneficiarias y las evaluaciones de las(os) agentes del programa.

Entrevistadora: —¿Cuándo empezaste a participar del Programa?

Beneficiaria: —Cuando estaba Silvia (una agente estatal), pero yo ya me había anotado y estuve como 7 meses sin cobrar. Yo ya estaba embarazada y cobré recién cuando mi hija ya tenía como un mes.

Entrevistadora: -Y ahí cuando fuiste y hablaste con las operadoras, ¿qué les contaste como para que te admitieran?

Beneficiaria: -Y que yo estaba peleada, no tenía a nadie.

Entrevistadora: $-¿$ Con quién estabas peleada?

Beneficiaria: - Con el papá de la nena, por boludeces, después hablamos y... ahí hablé con Silvia y otra chica más que estaba... la que me tomó la entrevista me dijo si yo estaba soltera $y$ le dije que sí, y que después que tenga la nena iba 
a buscar un trabajo, $y$ ellas me dijeron que me iban a aguantar a que yo consiga (Valeria, 20 años).

Este caso es típico porque representa la condición de vulnerabilidad, tal como fue enunciada por la agente estatal al definir la situación del riesgo para las chicas: el "estar solas". Esa condición bastaba y no requería ahondarse en si además de eso, existían o no vínculos con el delito. El programa no enuncia la posibilidad de ofrecer ayuda a varones que "estén solos", pero sí se muestra receptivo a que una chica que "está sola", reciba una ayuda de $\$ 150$ y contención afectiva o institucional.

Otro ejemplo puede ilustrarse con el caso de Leticia, beneficiaria de 25 años y madre de una niña. Leticia volvió al programa después de bastante tiempo (se había ausentado sin explicaciones y se le había dejado de pagar la ayuda económica). Cuando se presentó, contó que se le había terminado el trabajo temporal que tenía y que aunque con la plata que traía el marido de las changas que hacía "se arreglaban”, ella podía volver a venir. Además, estaba nuevamente embarazada y el plan de ayuda económica provisto por el programa le permitiría completar los ingresos familiares. Automáticamente, se le volvió a dar de alta, sin realizarse con ella ningún acuerdo personal (volver a la escuela, tomar un curso de formación, buscar trabajo fuera del hogar).

Sin delitos asociados y con un marido que hacía changas, ¿cuál era la necesidad que el programa consideraba legítima? $\mathrm{Ni}$ siquiera estaba "sola", en los términos en que el programa define tal situación. Este ejemplo insinúa que la "soledad" de las chicas podría estar más asociada a una condición intrínseca de las madres que viven en contextos desaventajados, que a la compañía o no de un varón de carne y hueso.

El trabajo de campo permitió advertir que la condición materna era, no solo la llave de acceso, sino la razón que las exceptuaba de cumplir con las contraprestaciones que el programa tenía previstas para sus beneficiarios(as) por cobrar el plan de ayuda económica (continuar los estudios, formarse en un oficio, resolver causas judiciales pendientes, tratar la adicción a las drogas, etc.). Para las chicas, cuidar de sus hijos parecía ser un compromiso válido no enunciado formalmente.

El caso de Silvina puede ilustrar esta afirmación. Ella, de 28 años, con 6 hijos, pero no todos a cargo, fue una de las que aceptó la propuesta del programa de volver a la escuela como contrapartida del cobro de la ayuda económica. Lo haría en horario nocturno y como no sabía leer ni escribir, la mandaron a segundo grado. Los primeros días de clases fue a la escuela con su hija de 2 años porque no tenía con quien dejarla; el malestar de la maestra por la presencia de la chiquita, sumado a que Silvina no entendía mucho la clase, la decidió a pasarse a primero, donde el nivel era más básico y la docente más comprensiva. El inicio, aunque con sobresaltos, había estado bien; sin embargo, un par de meses más tarde Silvina ya no iba más a la escuela. Estaba nuevamente embarazada. Lo que le explicó a la agente es que al ir a la escuela en la noche, llegaba muy tarde a la casa, además, el marido cuando volvía de trabajar no tenía nada para cenar y que la nena más pequeña, como ella llegaba tan tarde, se iba a dormir sin comer. En este punto, que Silvina dejara la escuela significaba que no cumpliría con el compromiso personal que debía asumir para ser beneficiaria. No obstante, sus razones para dejar de estudiar fueron entendidas como válidas. Parecía lógico, a los ojos del programa, que con varios chicos a cargo y un embarazo a cuestas, la escuela implicara demasiado esfuerzo. Silvina recibió las felicitaciones del caso por el embarazo y siguió "contraprestando" a través de su rol de madre y buena esposa.

Es más, como las chicas sostenían que necesitaban más ingresos pero que no podían conseguir trabajos fuera de sus casas por su responsabilidad materna, el programa incorporó variantes en su implementación para adaptar su oferta a las necesidades de las chicas. Habilitó un espacio de formación y reunión en el marco del programa para que las chicas que quisieran organizaran un microemprendimiento. Idealmente, así podrían obtener ingresos sin descuidar a sus niños. 
Del delito, en un programa que intenta prevenirlo, ni noticias. Estos casos dan la pauta de que el programa es comprendido por las beneficiarias como una suerte de compensación económica por cuidar a sus hijos y no poder trabajar fuera de sus casas. La persistencia de ellas en el dispositivo demuestra que el programa adhiere a esa lectura $y$ valida las necesidades expresadas por las chicas.

\section{DISCUSIÓN}

En otro trabajo (Medan, 2011) se detalló cómo el "proyecto de vida alternativo" para los beneficiarios de este programa, legitima un modelo de varón proveedor que obtenga ingresos legalmente, evite comportamientos riesgosos, irracionales e infantiles como el delito y sus acciones asociadas, $y$ que privilegie oportunidades de capacitación para proyectar un futuro deseable. Con base en este modelo, el programa -en ocasiones con la colaboración de los propios beneficiarios - refuerza estereotipos de masculinidad al naturalizarlos. Sin embargo, en relación con la dimensión generacional, la disputa es más cruda. El programa reprende a los varones por su rebeldía y los insta a "madurar", al tiempo que deslegitima las interpretaciones que ellos tratan de imponer sobre sus modos de actuar. Por ello, se sostiene que el programa subordina a los varones infantilizándolos y se ubica en la vereda de la adultez para legitimar el modelo de transición deseable que propone.

La dinámica de subordinación de las chicas se presenta de un modo mucho más sutil y quizás por ello, más profunda. Como la mayoría de las chicas se presenta al programa, no como delincuentes sino como madres pobres ${ }^{10}$ y la maternidad se reconoce como una práctica responsabilizadora que supone madurez, el programa considera válido el proyecto de maternidad. Así, ellas dejan de ser ubicadas en una posición inmadura como la juventud, si

$10 \quad$ Esta cuestión habilita otra discusión - que no se aborda en este artículo- sobre en qué medida la creciente admisión de chicas madres cuestiona la especificidad del objetivo del programa, prevenir el delito o al menos no invita a discutir más ampliamente los factores de riesgo o condicionantes para el delito. esta se caracteriza por la falta de un proyecto de vida. No es necesario re-encauzar a las chicas, solo requieren acompañamiento y colaboración con la ejecución del proyecto. A las chicas no se les pide madurar, ni se les exige estudiar ni trabajar, porque - lógicamente- bastante trabajo tienen con sus hijos(as). Parece que las chicas han completado una transición generacional deseable al momento de convertirse en madres, dejando atrás la juventud descarriada y problemática. El conflicto generacional entre la institución (adulta) que se propone transformar y conducir la transición generacional pierde relevancia. Sin embargo, al legitimar la maternidad como proyecto de vida se producen acciones colaterales y significativas que condicionan el ejercicio de la juventud y la feminidad.

En primer lugar, se invisibiliza - por contraste con la notoriedad de la maternidadla relación de las chicas con el delito. Como sugiere Elizalde, habrá que preguntarse si esa invisibilidad constituye un dato de la realidad pasible de verificación o de:

... un efecto de lectura de una construcción ideológica específica que "borra" a las mujeres de cierto ámbito de la "peligrosidad" para fijarlas en el campo de la "desviación sexual", la "vulnerabilidad" y el "riesgo social", y operar, desde allí, una regulación diferencial de sus prácticas (Elizalde, 2010).

La operación tiene un doble resultado porque al destinarlas a ámbitos privados y menospreciar su capacidad disruptiva, engrandece la de los varones. Es decir, como el género es un sistema de diferencias, al gestar una imagen de las chicas alejadas de las prácticas delictivas o arriesgadas, habilita a los varones a que ocupen esos lugares, contribuyendo a su vez a reforzar los estereotipos que los vinculan naturalmente con dichas acciones.

En segundo lugar, al tiempo que el programa excusa a las chicas de tareas que sí les exige a sus pares varones (conseguir un trabajo, retomar los estudios, etc.) y legitima de ese modo sus necesidades (como madres), las confina a esos espacios domésticos, no remunerados $y$ menos jerarquizados que a los que 
acceden los varones. Adhiere así a una mirada tradicional que indica que las mujeres deben permanecer al cuidado de sus hijos. En este sentido, el programa puede catalogarse como una iniciativa consciente de género, pero neutral en tanto reconoce las diferencias de género, pero no cuestiona las desigualdades entre mujeres y varones (Kabeer, 1994). La validación de la maternidad que se ha advertido en el trabajo de campo obstruye - por omisión- otras posibilidades de conciliar la feminidad, la familia y la obtención de ingresos propios.

Una tercera consecuencia es que al asistirlas porque "están solas" refuerza la idea de que las mujeres suelen definirse con base a sus relaciones con hombres, como esposas, madres, hijas o hermanas; en contraste con lo que ocurre con las distinciones correspondientes para los hombres: las mujeres no son determinantes para definir la masculinidad (Ortner y Whitehead, 2000). Por otro lado, más sutilmente, su condición de "soledad" parece intrínseca a su condición de madres pobres, ya que el programa las asiste aunque tengan parejas: el programa siempre las reconoce vulnerables y cumple el rol proveedor que no cumplen esos varones, o que no cumplen bien. Este procedimiento las señala como mujeres carentes, siempre necesitadas de ayuda de alguien o algo que se coloque en la posición de varón proveedor.

En síntesis, las chicas jóvenes merecen ayuda, no por "rebeldes" — como los varonessino por "débiles".

Sin embargo, ellas reconocen las asociaciones tradicionales entre feminidad $y$ vulnerabilidad, e independientemente de que se sientan reflejadas, hacen un uso estratégico de esta "debilidad" que se les impone como marca y se presentan en nombre de dicha interpelación.

Según Valeria Llobet, estas operaciones de apropiación y reutilización se despliegan como fruto de una "hibridez de los enunciados, que combinan la interpretación propia con un discurso institucional" (2009: 250). En una dirección similar, Silvia Elizalde ratifica la existencia de respuestas subalternas a las interpelaciones institucionales, que dan cuenta de aquella conformación relacional que supone la configuración de identidades atravesadas por el género, la clase y la edad. La autora explica que:

... los posicionamientos discursivos relevados en entrevistas a mujeres jóvenes de sectores populares van desde la acomodación estratégica a las identidades restrictivas construidas en su entorno, hasta la ruptura paródica y la inversión de la carga peyorativa por la autoafirmación, la discontinuidad subversiva o la disonancia más o menos radical entre la juventud "deseable", las prescripciones históricas sobre los géneros y la propia construcción identitaria (Elizalde, 2010).

De esta manera $y$ posiblemente, como fruto de sus anteriores vínculos con la "ayuda" estatal, las chicas han aprendido a presentarse como merecedoras de asistencia en pos del cuidado de sus hijos(as). Las interpretaciones que hacen sobre su maternidad conforman las expectativas de los programas y por ello, les permiten acceder a los beneficios y negociar condiciones. Sin embargo, la utilización estratégica que las chicas pueden hacer de su maternidad no logra neutralizar, ni mucho menos subvertir, su multideterminada posición subordinada.

\section{CONSIDERACIONES FINALES}

En su interés por garantizar el control $y$ la reproducción social, e incluso el bienestar, los programas estatales señalan transiciones legítimas e ilegítimas hacia la adultez y diferenciadas según género, que impactan en la vida cotidiana de los y las jóvenes. Observar los cruces entre la dimensión etaria y de género en la construcción de beneficiarios y beneficiarias de programas sociales es un modo de reconstruir las transiciones "deseables" entre la juventud y la adultez diferenciadas según género. Pero también permite enfocarse en los modos de contribuir a la configuración de identidades de género y etarias en el presente. Lo que en este artículo se pretendió exponer es, para el caso de las chicas, argumentos para ambos procesos. La transición deseable hacia la adultez para las chicas jóvenes aparece vinculada a un proyecto 
de maternidad que las incluye socialmente, pero desde una posición subordinada a la ayuda masculina o estatal. A su vez, ese mismo proyecto de inclusión - al que ellas prestan complicidad más o menos estratégicamente- les sugiere modelarse según una versión femenina adulta que limita — en la mayoría de los casos- sus capacidades disruptivas y sus despliegues públicos y autónomos.

\section{BIBLIOGRAFÍA}

\section{LIBROS}

Bourdieu, Pierre. Sociología y cultura. México: Grijalbo-CNCA, 1990.

Elizalde, Silvia. La otra mitad. Género y pobreza en la experiencia de mujeres jóvenes. La Plata: EdunLP, (en prensa).

Faur, Eleonor. Masculinidades y desarrollo social. Las relaciones de género desde las perspectivas de los hombres. Bogotá: UNICEF/Arango Editors, 2004.

Kabeer, Naila. "Gender-Aware policy and planning: a social-relations perspective". Gender Planning in Development Agencies. Meeting the Challenge. Macdonald, M. (ed.). Oxford: Oxfam, 1994.

Llobet, Valeria. ¿Fábricas de niños? Las instituciones en la era de la infancia. Buenos Aires: Novedades Educativas, 2009.

Ortner, Sherry y Whitehead, Harriet. "Indagaciones acerca de los significados sexuales". El género. La construcción cultural de la diferencia sexual. Lamas, Marta (comp.). México: PUEG, 2000.

Scott, Joan. "El género: una categoría útil para el análisis histórico". El género. La construcción cultural de la diferencia sexual. Lamas, Marta (comp.). México: PUEG, 2000.

\section{REVISTAS}

Chaves, Mariana. "Juventud negada y negativizada: representaciones $y$ formaciones discursivas vigentes en la Argentina contemporánea". Última Década 13 (23). 2005: 9-32.
Fraser, Nancy. "La lucha por las necesidades: esbozo de una teoría crítica socialista-feminista de la cultura política del capitalismo tardío". Debate Feminista. 1991.

Haney, Lynne. "Homeboys, babies, men in suits: the state and the reproduction of male dominance". American Sociological Review 61 (5). 1996.

Medan, Marina. "Sociabilidad juvenil masculina $y$ riesgo: discrepancias $y$ acuerdos entre un programa de prevención del delito juvenil y sus beneficiarios". Última Década 19 (35). 2011.

Medan, Marina. "¿Proyecto de vida? Tensiones en un programa de prevención del delito juvenil". Revista Latinoamericana de Ciencias Sociales, Niñez y Juventud. Universidad de Manizales, CINDE, 2012.

TESIS

Medan, Marina. "Construcciones de lo masculino y lo femenino en políticas sociales dirigidas a jóvenes. El caso del Programa Comunidades Vulnerables en la localidad de Avellaneda, 2008-2009". [Tesis de Maestría en Políticas Sociales]. Universidad de Buenos Aires, 2010.

OTROS

Dirección Nacional de Política Criminal (DNPC). Plan Nacional de Prevención del delito. Programa Comunidades Vulnerables. Buenos Aires: COGCAL, 2007.

Guzmán, Virginia. "Género en las políticas públicas”. Seminario Sistema de Equidad de Género en el programa de Mejoramiento de la Gestión, 2002.

Medan, Marina. "Registros de campo: $\mathrm{n}^{\circ}$ $48(26 / 11 / 08), n^{\circ} 57$ (11/03/2009), $\mathrm{n}^{\circ} 70(17 / 06 / 2009) "$. Investigación doctoral Prevención del delito con transferencias condicionadas de ingresos: negociaciones entre un programa social y jóvenes de sectores populares. Financiada por el CONICET- 
Argentina, mediante su programa de becas doctorales de "tipo I" (01/04/2008 a 31/03/2011 -resolución $\left.n^{\circ} 29 / 08\right)$ y "tipo II" (entre 01/4/2011 y el 31/03/2013 -resolución n 451/11).

\section{ENTREVISTAS}

Agente encargada de la implementación estudiada, barrio del Gran Buenos Aires donde se implementaba el programa estudiado, 30/04/08

Operadora comunitaria de la implementación estudiada, barrio del Gran Buenos Aires donde se implementaba el programa estudiado, 25/06/08

Victoria, beneficiaria del programa, barrio del Gran Buenos Aires donde se implementaba el programa estudiado, 01/10/08

Valeria, beneficiaria del programa, barrio del Gran Buenos Aires donde se implementaba el programa estudiado, 08/10/08

Fecha de ingreso: 10/04/2012 Fecha de aprobación: 30/05/2012 
